\title{
Monitoring of Patients Treated with Radiotherapy for Cervical Cancer
}

\section{Nkoua Epala $\mathrm{B}^{1 *}$, Bolenga Liboko $\mathrm{AF}^{4}$, Bintsené Mpika $\mathrm{G}^{2}$, Mbongo $\mathrm{A}^{2}$, Ndounga $\mathrm{E}^{4}$, Ngatali $\mathrm{SF}^{4}$, Moyikoua $\mathrm{R}^{3}$ and Nsondé Malanda $\mathrm{J}^{4}$}

${ }^{1}$ Radiotherapy Service, CHU Brazzaville, Republic of the Congo

${ }^{2}$ Gynecology-Obstetrics Department, CHU Brazzaville, Republic of the Congo

${ }^{3}$ Radiology Department, CHU Brazzaville, Republic of the Congo

${ }^{4}$ Medical Oncology Department, CHU Brazzaville, Republic of the Congo

*Corresponding Author: Nkoua Epala B, Radiotherapy Service, CHU Brazzaville, Republic of the Congo.
Received: May 13, 2021

Published: May 26, 2021

(C) All rights are reserved by Nkoua Epala B., et al.

\section{Abstract}

Cervical cancer is the second most common female cancer after breast cancer in developing countries including Congo. Patients are diagnosed in most cases in the advanced stages and are treated in a standard way by concomitant radio chemotherapy.

After the treatment of cervical cancers by radiotherapy, surveillance is an important stage in life after irradiation because it can either detect and treat the late effects of irradiation, detect and treat early a possible tumor recurrence, or reassure patients of the possibility of complete remission or cure. Radiation monitoring is clinical and radiological.

It was a good opportunity for us to take stock of the surveillance of patients with radiotherapy at the University Hospital Centre (CHU) in Brazzaville and abroad for cervical cancer.

Keywords: Surveillance; Radiation Therapy; Cancer; Breast; Treatment

\section{Introduction}

Cervical cancer is the second most common cancer after breast cancer in the Congo, according to the Brazzaville Cancer Registry. The majority of patients are seen at the locally advanced stage whose therapeutic gold standard remains to this day concomitant radio chemotherapy (RCC). After the treatment of cervical cancers by radiotherapy, surveillance is an important stage in life after irradiation because it can either detect and treat the late effects of irradiation, detect and treat early a possible tumor recurrence, or reassure patients of the possibility of complete remission or cure. Monitoring after radiotherapy is clinical and radiological. It was a good opportunity for us to take stock of the surveillance of patients with radiotherapy at the University Hospital Centre of Brazzaville (CHUB) and abroad for cervical cancers.

\section{Patients and Methods}

We undertook a descriptive retrospective study in the hersic radio and medical oncology department of the University Hospital of Brazzaville (CHUB), in January 2014 and December 2020. To be included in the study, patients had to meet the following criteria: having histological confirmation of the diagnosis of cervical cancer; Have received radiation therapy at THE CHUB or abroad.

Have a medical report confirming the effectiveness of radiation therapy; Have medical imaging records of the various controls. The 
patients were referred for radiotherapy by the medical oncology, gynecology and obstetrics and radiology departments of the Brazzaville University Hospital.

All patients had received radiotherapy at the CHUB or abroad and had a medical report signed by a radiotherapist. For patients treated at the Brazzaville Iversitary Hospital, the radio Thetis received was conventional cobaltherapy with a Thermatron $780 \mathrm{C}$ brand device. This device is equipped with a Radioactive Cobalt 60 source emitting gamma radiation with an average energy of 1.25 $\mathrm{MeV}$. Treatment with external radiotherapy took place in two stages: the first simulation and the treatment itself afterwards.

During the simulation, the patients were positioned in dorsal decubitus and well aligned by the lasers under the machine. The circumventing of the target volumes was carried out according to the anatomical bone markers using standard images of the pelvis. $\mathrm{X}$-rays of the pelvis to all patients had helped to delineate the fields of irradiation, to draw the protective caches of the so-called at-risk organs namely the bladder, rectum, femoral heads and intestinal handles. This imaging also provided the data needed to achieve dosimetry.

During the treatment phase, all patients received the total dose of 66 Grays in 33 sessions in four beams with a conventional split of 2 Grays per session in two phases. In the first phase, patients received 46 Grays in 23 sessions. The absence of brachytherapy in our service explains the supplement of the dose by external radiotherapy. Thus, in the second phase the patients received 20 Grays in 10 sessions. The patients had received 5 sessions per week with an average spread of 46 days. However, other patients treated abroad had received conformational radiotherapy with a linear particle accelerator. They had received a total dose of 70 grays in 35 sessions at a rate of 2 grays per fraction with or without boost (irradiation supplement) on the parameters and pelvic or low back ganglions. The split was classic ( 5 weekly sessions of 2 grays per session). The radiotherapy monitoring schedule follows certain rules governed by learned societies and radiotherapy repositories.

After irradiation for cervical cancer with or without concomitant chemotherapy, patients are consulted immediately within the week that concludes the radiotherapy sessions to establish the end-of-treatment medical report and explain the pace of the checks for the next five years.
Patients will be reviewed by the radiation oncologist every three months for two years and then every six months for three years, and finally annually beyond five years of follow-up. This over-examination is clinical and radiological.

Clinically, it is a matter of thorough examination and clinical examination to look for signs of a possible recurrent local cervical, a new suspicious lesion of recent onset, but also to detect, diagnose and treat the onset of acute or late side effects of radiotherapy.

Biologically, no tumor markers have been validated to date.

From a radiological point of view, there-conducting of examinations is not systematic.

In case of call signs and depending on the suspected organ, magnetic resonance imaging (MRI) is the first recommended examination to better explore the pelvis.

In our context where MRI is not always available and accessible to all, we often limit ourselves to performing a CT scan, if not an ultrasound. In general, the examinations after radiation therapy for breast cancer will take place at least three months or so near the end of radiation therapy, followed by the standard follow-up schedule in radiotherapy. The Graph pad Prism 5 software was used to calculate the spread and compare our data with those of the literature.

\section{Results}

During the study period, 68 patients treated with radiotherapy for cervical cancers were recorded. The average age was 54 (extreme: 34 - 78 years). The most common histological type was invasive epidermoid carcinoma. Patients were classified according to the FIGO classification (Table 1). Acute side effects after irradiation were observed in 26 patients, while late side effects beyond six months after heralded radio were employment in 32 patients. The different types of side effects observed during surveillance are illustrated in figure 1 and 2 and the therapeutic response observed and the examinations performed during the course of the course are illustrated in figure 3.

Patient monitoring was carried out according to the standard follow-up schedule for patients treated with radiotherapy. 


\begin{tabular}{|l|c|c|c|c|c|c|}
\hline $\begin{array}{c}\text { Age } \\
\text { ranges }\end{array}$ & IIA2 & IIB & IIIA & IIIB & VAT & Total \\
\hline $30-40$ & & 3 & 1 & 1 & & 5 \\
\hline $41-50$ & 1 & 6 & 3 & 3 & 2 & 15 \\
\hline $51-60$ & 2 & 2 & 8 & 14 & 6 & 32 \\
\hline $61-70$ & & 2 & 4 & 6 & 2 & 14 \\
\hline $71-80$ & & & & 1 & 1 & 2 \\
\hline Total & 3 & 13 & 16 & 25 & 11 & 68 \\
\hline
\end{tabular}

Table 1: Patient breakdown by age and stage of extension (FIGO).

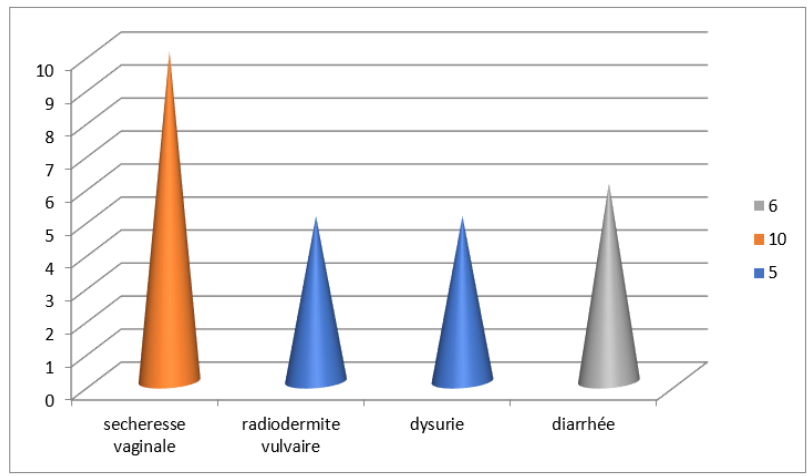

Figure 1: Acute side effects during surveillance.

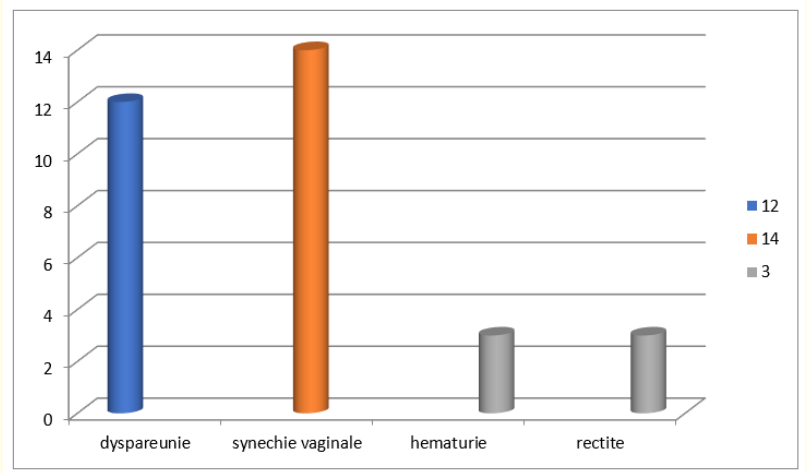

Figure 2: Late side effects during surveillance.

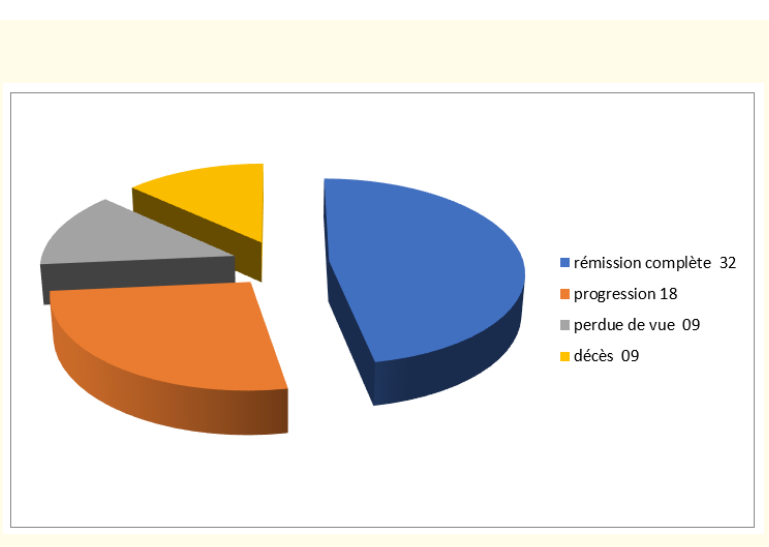

Figure 3: Therapeutic response observed during surveillance.

\section{Discussion}

After radiation therapy for cervical cancer, it is essential to have regular follow-up to ensure that the disease does not recur. Regular monitoring can also be an opportunity for women to report to the medical profession the difficulties encountered in their postcancer life, and to address sensitive topics such as sexuality or psychological disorders.

Several randomized clinical trials have shown that concomitant radio chemotherapy (RCC) has become the gold standard in the treatment of locally advanced cervical cancers. This therapeutic modality is superior to exclusive radiotherapy in terms of local control, recurrence-free survival and overall survival [1]. Indeed, the success of radiotherapy depends mainly on the total dose delivered in a homogeneous manner at the level of the tumor because it will ensure local control but also the protection of the so-called at-risk neighbourhood organs. However, the delivery of this dose is limited by the tolerance of healthy tissues in the volume irradiated.

The preservation of the quality of healthy tissues is therefore a major concern for the radiotherapist and must be integrated into the daily management and follow-up of patients. The deleaness of the onset of secondary lesions is a function solely of the life of mature cells. The duration of functional recovery is determined by the severity of stem cell depletion, itself resulting from the dose received [1,2]. Early reactions appear in fast-renewing tissues such as the skin (epithelite) and the mucous membrane (mucite). 
If the dose of irradiation is high enough to kill all stem cells, cell regeneration depends on the migration possibilities of stem cells from adjacent, unradiated tissue regions. In this case the volume or the irradiated surface influences the severity and duration of acute toxicities $[4,6]$. During the treatment period, side effects such as vulvo-vaginal and rectitis radio epi epi epithelites, as well as dermatitis in the gluteal fold, were observed in almost all irradiated patients.

Acute side effects during irradiation and in the first three months of the treatment were observed in 26 patients. However, late side effects occurring after three months of the end of the herald radio were observed in 32 patients.

Other side effects described in the following literature can be observed: vaginal dryness, dyspareunia, synechia, vaginal fibrosis, etc. Cicatrisation occurs by re-epithelizing from the surviving cell islands of the basal layer. Early lesions of the epidermis are low in dose per session but are highly influenced by sprawl due to cell repopulation.

Treatment of acute side effects depends on the grades or extent of the lesion. For example, for grade 1 and 2 lesions, the application of watery Eosine is recommended after the radiation therapy session and then in the evening at bedtime. All patients irradiated in our series with acute side effects were treated with watery Eosine. The acquisition of a linear accelerator to replace the cobaltherapy device at the University Hospital Center of Brazzaville, will certainly minimize the acute toxicities found in most of our patients and improve the quality of life of our irradiated patients [1-24].

\section{Conclusion}

Radiation therapy remains central to strategies for managing invasive cervical cancers. Monitoring after pelvic irradiation is based on French and international recommendations. It includes early assessment of post-treatment response, as well as the search for local or remote recurrences that are available for remedial therapies. Follow-up should also look for possible digestive, urinary or genital and sexual toxicities, which may have an impact on quality of life.

\section{Bibliography}

1. Barillot I., et al. "Complications of treatment of invasive cervical cancers on intact uterus. Results andprevention". Cancer/ Radiotherapy 4 (2000): 147-158.
2. Davidson SE., et al. "The impact of radiotherapy for carcinoma of the cervix on sexual function assessed using the LENT-SOMA scales". Radiotherapy Oncology 68 (2003): 241-247.

3. Ngomo KMJ., et al. "Evaluation of cervical cancer management by tele-cobalt at the Libreville Hospital Center". Médecine d'Afrique Noire 5111 (2004): 579-584.

4. Ozsaran Z., et al. "Radiochemotherapy for patients with locally advanced cervical cancer: early results". European Journal of Gynaecological Oncology 24.2 (2003): 191-194.

5. Jensen PT., et al. "Longitudinal study of sexual function and vaginal changes after radiotherapy for cervical cancer". International Journal of Radiation Oncology • Biology • Physics 56 (2003): 937-949.

6. Caffo 0., et al. "Physical side effects and quality of life during postoperative radiotherapy for uterine cancer. Prospective evaluation by a diary card". Gynecologic Oncology 88 (2003): 270-276.

7. Seiwert TY., et al. "The concurrent chemoradiation paradigmgeneral principles". Nature Reviews Clinical Oncology 4.2 (2007): 86-100.

8. Barillot I and Haie-Meder C. "Association of radiotherapy and chemotherapy in gynecological cancers". Bulletin Cancer 96 (2009): 271-283.

9. Barbera L and Thomas G. "Management of early and locally advanced cervical". Seminar on Oncology 36 (2009): 155-169.

10. Joly-Lobbedez F. "Concomitant chemotherapie in cervical cancers: what levels of evidence?" Cancer/Radiotherapie 13 (2009): 503-506.

11. Green JA., et al. "Survival and recurrence after concomitant chemotherapy and radiotherapy for cancer of the uterine cervix :a systematic review and meta-analysis". Lancet 358 (2001): 781-786.

12. Lukka H., et al. "Concurrent cisplatin-based chemotherapy plus radiotherapy for cervical cancer - a meta analysis". Clinical Oncology 14 (2002): 203-212.

13. Sheu MH., et al. "Preoperative staging of cervical carcinoma with MR imaging: a reappraisal of diagnostic accuracy and pit- 
falls". European Radiology 11.9 (2001): 182833.

14. Sala E., et al. "The added role of MR imaging in treatment stratification of patients with gynecologic malignancies: what the radiologist needs to know". Radiology 266.3 (2013): 71740.

15. Sahdev A., et al. "The performance of magnetic resonance imaging in early cervical carcinoma: a long-term experience". International Journal of Gynecological Cancer 17.3 (2007): 62936.

16. Jeong BK., et al. "Indications for endoscopy according to the revised FIGO staging for cervical cancer after MRI and CT scanning". Gynecologic Oncology 23.2 (2012): 805.

17. Loft A., et al. "The diagnostic value of PET/CT scanning in patients with cervical cancer: a prospective study". Gynecologic Oncology 106.1 (2007): 2934.

18. Badoual C., et al. "[HPV (Human Papilloma Virus) involvement in other cancers than gynaecological]". Rev Internal Medicine Founded by Natl French Society Internal Medicine 36.8 (2015): 5407.

19. Mota F. "Microinvasive squamous carcinoma of the cervix: treatment modalities". Acta Obstetricia et Gynecologica Scandinavica 82.6 (2003): 5059.

20. Lécuru F., et al. "Bilateral negative sentinel nodes accurately predict absence of lymph node metastasis in early cervical cancer: results of the SENTICOL study". Journal of Clinical Oncology 29.13 (2011): 168691.

21. Kadkhodayan S., et al. "Sentinel node biopsy for lymph nodal staging of uterine cervix cancer: a systematic review and metaanalysis of the pertinent literature". European Journal of Surgical Oncology 41.1 (2015): 120.

22. Zhou J., et al. "Robotic vs laparoscopic radical hysterectomy for cervical cancer: a meta-analysis". International Journal of Medical Robotics and Computer Assisted Surgery 30 (2015).

23. Ramirez PT., et al. "Management of low-risk early-stage cervical cancer: should conization, simple trachelectomy, or simple hysterectomy replace radical surgery as the new standard of care?" Gynecologic Oncology 132.1 (2004): 2549.
24. Ngô C., et al. "Outcome in early cervical cancer following preoperative low dose rate brachytherapy: a ten-year follow up of 257 patients treated at a single institution". Gynecologic Oncology 123.2 (2011): 24852.

\section{Volume 5 Issue 6 June 2021}

(C) All rights are reserved by Nkoua Epala B., et al. 